\title{
COVID-19: Pitfalls in Offering Research Participation as Therapy in Clinical Settings
}

\author{
Shivakumar Narayanan, MBBS, MD ${ }^{7}$, Joel V. Chua, MD , and Emily Heil, PharmD² \\ 'Institute of Human Virology, University of Maryland School of Medicine, Baltimore, MD, USA; ${ }^{2}$ University of Maryland School of Pharmacy, Baltimore, \\ MD, USA.
}

J Gen Intern Med 35(1 1):3359-60

DOI: $10.1007 / \mathrm{s} 11606-020-06158-6$

(c) Society of General Internal Medicine 2020

\section{INTRODUCTION}

As the coronavirus disease-19 (COVID-19) pandemic rages on through its seventh month, the USA bears the largest burden of the over 15 million cases worldwide. ${ }^{1}$ Events of an epidemic unfold through progressive revelation, to managing randomness, and finally negotiating a public response. ${ }^{2}$ In the earlier phases of the pandemic, clinicians were venturing to treat COVID-19 patients with therapies of unproven efficacy based on data from poorly conducted clinical trials and observational and anecdotal data. With limited peer-reviewed prospective data on therapies with mortality benefit, guidelines published since by societies have chosen to rein in the randomness by not recommending specific antiviral or immunebased therapy outside the setting of a clinical trial. Offering research participation as therapy represents a disadvantage for communities or populations who may have limited access to clinical trials or not be suited to participate in clinical trials.

\section{ACCESS TO CLINICAL TRIALS IS NOT WIDESPREAD OR UNIFORM}

To date, there are 170 phase 3 clinical interventional COVID19 studies registered on Clinicaltrials.gov that are currently recruiting patients. These would offer therapeutic interventions of potential benefit only to a small subset of subjects with COVID-19 at upper accrual limits. Additionally, some of these trials may be studying supplemental treatment modalities and not evaluating clinically relevant endpoints.

Black communities and communities of color bearing a disproportionately high burden of COVID-related morbidity and mortality with more than threefold higher infection rates and sixfold higher death rates, ${ }^{3}$ yet may be unable to access effective care in a timely manner due to barriers to care access. Racial and ethnic minorities remain underrepresented in clinical trials due to lack of awareness, trust or comfort with the

Received July 23, 2020

Accepted August 14, 2020

Published online September 3, 2020 process, and resource constraints. Subjects with lower income consistently have lower clinical trial participation.

Clinical trials may have rigorous exclusion criteria and comorbidities including chronic kidney disease which may have higher prevalence in the African American population or chronic liver disease in the American Indian or Asian population may exclude them from clinical studies. Residents of medically underserved communities and rural communities have limited access to healthcare and clinical trials. ${ }^{4} \mathrm{Commu}-$ nity hospitals and rural hospitals, which may provide vital safety net access to care, may not be able to offer access to research studies due to lack of research staff.

\section{RESEARCH PROCESS DIFFICULTIES EXIST AND MAY EXCLUDE SPECIFIC GROUPS UNFAIRLY}

Health literacy and language barriers for subjects who need an interpreter to communicate may lead to the unfair exclusion of such candidates, especially if they are hospitalized under enhanced isolation precautions which makes the informed consent process exceedingly difficult. Similarly, persons infected with COVID-19 who are incarcerated may not be selected due to their classification as a vulnerable population, or the inability of the study team to follow up for subsequent prespecified encounters post-discharge. This is unfortunate in the face of multiple outbreaks of COVID-19 in prisons across the country.

Hospitalized patients may be under significant mental stress, while in complete isolation from families or other sources of support. Physician contact is also often limited and through audiovisual media and may not help patient confidence.

Impaired decision-making in a hospitalized patient with COVID may be worsened by hypoxia, anxiety, and medical therapies, in addition to potential exacerbation of underlying comorbidities. The stress of hospitalization and the relatively lack of privacy or autonomy enjoyed in health may cause patients to adopt a passive role to suggestion in the medical decision-making process. ${ }^{5}$ They may be additionally tasked with making decisions regarding advance care planning. When faced with the prospect of their own mortality, severely ill patients may experience stress and related decisional 
conflict and may not be able to reasonably think through risks and benefits of participation in clinical trials.

Around $5-15 \%$ of patients with COVID infection may be intubated and on mechanical ventilation and may not be able to consent for a clinical trial. Substituted judgment by surrogates may not accurately reflect the patient's wishes especially in research which involves more than minimal risk. The socioeconomically disadvantaged or homeless may not have an available surrogate to consent.

\section{ATTITUDES TO RESEARCH VARY AMONG SUBJECTS AND PROVIDERS}

Additionally, decision-making may be anchored on availability and in a time of ever-changing available information about COVID-19, accessible through social and popular media, background knowledge and hearsay may play a role more than ever. Perceptions of the status of personal health or estimation of efficacy of medication may be inaccurate and may be influenced by portrayals of extreme outcomes in media. Even for treating clinicians, the deluge of clinical information through various sources has made it difficult to stay up to date with newly available information. Literature on COVID-19 represents one of the biggest expanding accumulations of scientific information, doubling every 20 days with papers accessible through various sources, including preprint servers and social media. ${ }^{6}$ This faster dissemination of information has also meant that low-quality data, duplicated, and even inaccurate data have found its way into the discourse in the medical community. Differing scientific opinions from experts, changing trends, and reversals of management guidelines within institutions may leave clinicians with differing opinions on substantive issues, including potential therapies.

A patient may be eligible for multiple clinical studies being offered at an individual site and treating physicians may not have accurate and complete knowledge of risks and benefits of individual studies. Enrolling a patient in a research study may present a conflict for the treating physician between doing what they may believe is in the patient's best interest within the scope of the practice of medicine and being able to contribute to discovery and dissemination of potential new treatments through research. ${ }^{7}$ The moral dilemma of withholding what may be perceived as a potentially effective treatment to a subset of participants in a randomized trial in favor of placebo may affect the concept of therapeutic equipoise and may present obstacles to the completion of a controlled trial. Shared decision-making between physicians and patients may be suboptimal given goals of participation in trials would seem abstract and not immediate to the clinical picture. Patients who are critically ill may be disinclined to be altruistic. Participation in the study could be viewed as receiving an effective treatment option rather than seeking to study efficacy in a randomized clinical trial setting. The potential for therapeutic misconception - the inappropriate assumption in part of patients that therapy and research were governed by a common primary goal of advancing the individual patient's best interests-is high.

Researchers must recognize the importance of the grounding ethical principles of respect, beneficence, and justice when conducting human subject research. Conducting clinical research into therapies for COVID-19 while simultaneously ensuring the provision of optimal clinical care to ill patients creates uncomfortable intersections. It is of concern that while attempting to straddle both worlds, the principles of justice (through lack of access to clinical research) and respect for autonomy (through issues with the informed consent process) may not be adequately met. While there are some potential solutions from the clinical practice and research enterprises, ${ }^{7}$ many obstacles remain to making this a successful endeavor.

Corresponding Author: Shivakumar Narayanan, MBBS, MD; Institute of Human Virology, University of Maryland School of Medicine, Baltimore, MD, USA (e-mail: snarayanan@ihv.umaryland.edu).

\section{Compliance with Ethical Standards:}

Conflict of Interest: The authors declare that they do not have a conflict of interest.

\section{REFERENCES}

1. Johns Hopkins University Coronavirus Resource Center. Accessed 06/22/ 2020 at 1 PM. https://coronavirus.jhu.edu/map.html

2. Rosenberg CE. What is an epidemic? AIDS in historical perspective. Daedalus. 1989;118(2):1-17.

3. Yancy CW. COVID-19 and African Americans. JAMA. 2020;323(19):18911892. doi:https://doi.org/10.1001/jama.2020.6548

4. Dandachi D, Reece R, Wang EW, Nelson T, Rojas-Moreno C, Shoemaker DM. Treating COVID-19 in Rural America. J Rural Health. 2020

5. The kindly stress of hospitalization. Am J Med. 1985;78(4):A78-A90.

6. Scientists are drowning in COVID-19 papers. Can new tools keep them afloat? Jeffrey Brainard. Science Magazine.May 13,2020. Accessible at https://www.sciencemag.org/news/2020/05/scientists-are-drowningcovid-19-papers-can-new-tools-keep-them-afloat

7. Angus DC. Optimizing the Trade-off Between Learning and Doing in a Pandemic. JAMA. 2020; doi:https://doi.org/10.1001/jama.2020.4984.

Publisher's Note Springer Nature remains neutral with regard to jurisdictional claims in published maps and institutional affiliations. 